\title{
Analytical confidence and insomnia
}

\author{
"This article does not address statistical confidence. Rather, it discusses the risk of having an insufficiently selective analysis. It \\ is about having confidence that you are presenting reliable results to your peers."
}

As a young scientist, I remember dreaming of a perfectly specific analysis in which only my compound of interest was being measured. Some of my contemporaries still wake in the night worried about the quality of their data. With any analysis there will always be the risk of interference, but how can you minimize the risk in LC-MS analyses?

This article does not address statistical confidence. Rather, it discusses the risk of having an insufficiently selective analysis. It is about having confidence that you are presenting reliable results to your peers. It also addresses those experiments in need of the highest assurance of correct data: sport doping.

This article is separated into several parts: definitions, achieving more confidence via selectivity, an analogy to describe field asymmetricwaveform ion mobility spectrometry (FAIMS) separation, some concerns about this technology, the human characteristics that predispose a scientist to success with new technologies and the final section describes the value of keeping interferences out of the mass spectrometer.

\section{Definitions}

Specificity is generally defined as the measurement of the signal produced by the targeted analyte alone [1]. Selectivity is the measurement of the target analyte to the exclusion of most other compounds in the sample. A perfectly specific method may be obtainable but, like the uncertainty principle, if you have a specific method, you will never be able to prove it. Therefore, the best you can hope for is a highly selective method.

Sensitivity among bioanalytical scientists has many definitions. There are four types of sensitivity. For triple quadrupole MS users, instrument sensitivity often refers to the response for a given concentration infused. An alternative definition for MS sensitivity is ion-trap sensitivity. Ion-trap instruments are sensitive, but it comes at the price of time and is related to selectivity.
When the trap fills predominantly with the ion of interest (selectivity), it will take longer to fill, but the spectrum at the end will demonstrate very high sensitivity (response).

Calibration lines also display sensitivity. This definition is less commonly used but still valid. A sensitive calibration line suggests that a small change in the $\mathrm{x}$-dimension (prepared standard concentration) will result in a large change in the $y$-dimension (response, calculated concentration). In comparison, if the slope is near zero, then the calibration line may be said to be insensitive.

The fourth definition is assay sensitivity. It is a very practical definition and refers to the assay being described as 'fit for purpose.' Assay sensitivity really refers to the ability of the entire system (human operators and instrumentation) to present the supervisor with acceptable results within the limits of the analysis. If there are interferences, the assay characteristics change and the assay sensitivity decreases. Even with a very high signal-to-noise ratio, a very selective method may be obtained, but this still does not address the issue of the unresolved chromatographic or MS interferences.

\section{Achieving more confidence via selectivity}

Removing interferences increases the selectivity of the analysis and, therefore, increases confidence. Confidence can also be increased by tightly controlling experimental errors and then monitored by statistical analysis. Assuming this is completed with high accuracy and precision, what more can a scientist do to further increase confidence? You need more selectivity.

There are numerous techniques readily available to increase selectivity. In the case of the triple-quadrupole MS, higher mass resolution is one option. Another is the selection of an alternate transition for quantitation. In both cases, even though instrument sensitivity may

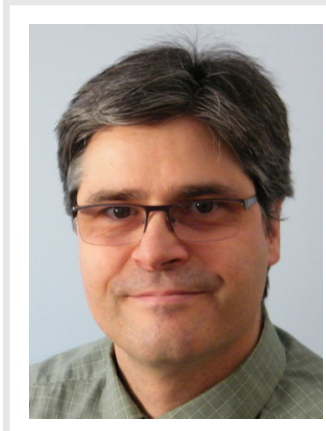

James Kapron

Thermo Fisher Scientific, 2845 Argentia Ave, Unit 4, Mississauga, ON, L5N 8G6, Canada Tel.: + 6133557117

E-mail: james.kapron@ thermofisher.com 
be reduced, signal-to-noise may be increased, providing a better overall result. In the case of chromatography, options include ultra-HPLC for increased chromatographic resolution or stationary phase changes to alter the column selectivity. With sharper chromatographic peaks and altered column selectivity there is a greater likelihood of the resolution of unwanted interferences but, unfortunately, simply applying these options does not always mean the selectivity has actually increased. The interferences may still be lurking beneath the analyte.

To improve selectivity, many bioanalytical scientists prefer to adjust the sample-preparation process. However, every additional manipulation introduces sample loss and the risk of user error. Some sample preparation must be performed, but overdoing the purification in the condensed phase provides diminishing returns.

These standard methods of improving selectivity (i.e., LC, MS and sample preparation) represent conventional methodologies: baby steps within the same well-defined comfort zone. An alternate dimension of selectivity may be obtained by exploiting a different physical attribute of the analyte.

Analogy to describe FAIMS separation Field asymmetric waveform ion mobility spectrometry works by ion mobility. Consider twins having leapt from an aircraft; both weigh the same, but they have parachutes of different sizes. As they drift through the atmosphere they do not reach the earth at the same time. But now consider two skyscrapers that apply an electric field on either side of the twins. As they fall and before they reach the ground the electric field pushes and pulls the twins to the left and right. The effective distance travelled is much greater via this zig zag pathway than by a simple linear fall. The longer distance allows for more opportunity to separate the twins from each other. In this analogy, one twin might be the target analyte and the other might be the interference: they possess the same $\mathrm{m} / \mathrm{z}$ but different flow characteristics through the gas.

The main reason to use gas-phase atmospheric pressure separation is because it is still comparatively new and yet sufficiently established that the early teething issues have been worked out of the technology. There is still ample room for publication of novel research. The more adventurous and skilled researchers, by developing a FAIMS method, gain a competitive advantage that other, less adept researchers cannot match.
For example, in the case of unscrupulous, doped athletes, it would be very satisfying to catch someone cheating at competitive athletics. New selectivity technologies are a great opportunity to extend the detection time post-dose and level the playing field among athletes [2].

\section{Concerns about FAIMS}

How much of a structural change will cause a significant separation? Cluster interferences typically are separated from small molecules. Drugs and their metabolites frequently emerge together. Doubly and triply charged tryptic peptides emerge in a band together, but are distinctly separated from singly charged ions. Thus, if the interferences are structurally unrelated to the analytes, they are frequently resolved using standard conditions. It is of particular note that because drugs and metabolites commonly emerge together, compensation voltage switching is not required with these multianalyte assays.

The modern FAIMS device uses helium and there is some concern about the cost of this noble gas. The most common way of reducing helium consumption is to use it only when the twins have jumped, not while the aircraft is taxiing for take off, reaching stable altitude or when the plane is landing.

\section{Human characteristics for success}

New technologies are risky, just like jumping from an airplane. Can we predict the personality characteristics that predispose a tendency to success with new selectivity technologies? The first of the two characteristics is ability. Pasteur has suggested that if your mind is prepared to see the connections, if you have the ability to learn and understand, then you are likely to succeed [3].

The second characteristic is an emotional predisposition, a genuine desire to learn and an internal drive to accomplish a goal. For example, are you trying to stay ahead of the competition? Are the stakes for success high? This is true in sport doping analysis, where unscrupulous chemists create new compounds that mask the anabolic steroids [4]. The good guys believe new selectivity techniques serve the world by keeping honest athletes honest.

Do you know a scientist with the personality characteristic of obsession with interferences? Are lawyers trying to work ways around their patents? If extra chromatographic peaks allow them to convince the law courts that your data are not reliable, it may be of value to have fewer - or no other - peaks in the chromatogram. 
Does the quality assurance group give you a headache about slightly quirky chromatographic peak shapes? In high-stake GLP laboratories, a failed analysis might give no result for critical samples. Another outcome of study inspection by a regulatory agency might force the repetition of the study. In serious cases, the withholding of GLP status for that laboratory due to incorrect pharmacokinetic data may give good reason to pursue increased selectivity.

\section{The value of keeping interferences out of the mass spectrometer}

A final word on what selectivity should provide. Traditionally, LC separates compounds and everything is introduced into the MS. Divert valves were invented so that involatile salts do not interfere with instrument robustness. The goal for other selectivity techniques should be similar to divert valves. Mass spectrometers are still very high-priced instruments, despite the gains of recent years. It will always be a bad idea to let everything into your MS. Do not junk it up with ions that are not of interest. Selectively introduce them and let the power of the combined selectivity techniques allow you to sleep better at night. FAIMS is another tool to give you confidence. Experimentally, you will wake up as if your previous troubles were a nightmare, when you realize the problem is solved.

\section{Financial \& competing interests disclosure}

The author is employed by Thermo Fisher Scientific, a manufacturer of field asymmetric waveform ion mobility spectrometry technology. The authors have no other relevant affiliations or financial involvement with any organization or entity with a financial interest in or financial conflict with the subject matter or materials discussed in the manuscript apart from those disclosed.

No writing assistance was utilized in the production of this manuscript.

\section{Bibliography}

1 Persson B-A, Vessman J. Generating selectivity in analytical chemistry to reach the ultimate - specificity. Trends Anal. Chem. 17(3), 117-119 (1998).
Guddat S, Thevis M, Kapron J, Thomas A, Schaenzer W. Application of FAIMS to anabolic androgenic steroids in sport drug testing. Drug Test. Analysis 1(11-12), 545-553 (2009).
3 Pasteur L. Dans les champs de l'observation le hasard ne favorise que les esprits préparés. In : Euvres de Pasteur (Volume 7). Pasteur Vallery-Radot L. (Ed.). Masson, Paris, France, 131 (1922-1939).

4 Rivier L. New trends in doping analysis. Chimia 56(3), 84-90 (2002). 